\title{
Lost world of complex life: Molecular traces of our deep eukaryotic ancestors
}

\author{
JOCHEN J. BROCKS ${ }^{1}$, BENJAMIN J. NETTERSHEIM ${ }^{1,2}$
}

${ }^{1}$ Research School of Earth Sciences, Australian National University (jochen.Brocks@anu.edu.au); ${ }^{2}$ Max-PlanckInstitute for Biogeochemistry (bnett@bgc-jena.mpg.de).

Little is known about our deep eukaryotic roots. Eukaryotes are organisms with complex cells that possess organelles such as a nucleus and mitochondria. The group includes animals, plants, fungi, algae and single celled protists. Based on molecular clock estimates, the last common ancestor of all living eukaryotes (LECA) roughly originated some 1 to 2 billion years ago. However, going deeper back in time, past LECA, the history of eukaryotes becomes obscure as there are no fossils that can be attributed to pre-LECA eukaryotes with confidence. A second line of enquiry to study ancient eukaryotes are steranes, the molecular fossils of eukaryotic sterols. However, the oldest clearly indigenous eukaryotic steranes only date back to $\sim 800 \mathrm{Ma}$. In this presentation we describe the hunt for elusive eukaryotic steranes in yet older sediments.

In 1964, Konrad Bloch won the Nobel Prize for deciphering the biosynthetic pathway of the sterol cholesterol. $\mathrm{He}$ found that cholesterol is produced from epoxysqualene in no less than 13 enzymatic steps. The biosynthesis of sterols is so intricate and energy intensive that their precise structure must be crucial for the eukaryotic cell. This insight led Bloch to the famous hypothesis that the biosynthetic pathway towards cholesterol was perfected in a Darwinian process step by step over hundreds of millions of years, and that each of the intermediates was an improvement over its precursor. At some point in deep time, each intermediate must have been a functional end-product in the membrane of a pre-LECA ancestor. Bloch's hypothesis predicts that the geological record may hide an array of fossil sterols (steranes) that record the sequence of assembly of the original biosynthetic pathway in deep time.

Contrary to the current paradigm, we show that fossil sterols are abundant in the sedimentary record between 1.6 and $1.0 \mathrm{Ga}$. The molecules had remained obscure as they possess unexpected structures. They comprise two successive biosynthetic steps in Bloch's pathway and may thus represent the first observation of evolutionary biosynthetic intermediates in the geological record, potential witnesses of Proterozoic oceans that were teaming with our most distant eukaryotic ancestors. 